\title{
SOME NEW RECTIFYING EFFECTS IN CONDUCTING GASES.
}

By P. G. Nutring.

The passage of electricity from a metal to a gas, or from a gas to a metal, occurs in a manner not yet well understood. The present investigation was undertaken to determine what conditions of the metal or gas favor the transfer of electricity from one to the other, and, if possible, to throw some light on the mechanism of surface conduction. While the results here presented are crude, they bear directly on the theory of metal-gas conduction, and the method, though very simple, is believed to be new. The work suggested itself during some spectroscopic work with Plücker tubes.

If we use a direct current in studying the electrode drop in potential, we are confronted by the the great difficulty in keeping the condition of the surface, temperature, pressure, etc., constant, and in reproducing these conditions in successive tests. But if we use an alternating current of a maximum voltage just a little above the maximum total drop in potential between electrodes, conditions can not appreciably change between successive alternations, and the excess of current in one direction may be taken as a rough measure of the relative surface conductivity at the two electrodes. It is hardly conceivable that there can be any rectifying effect due to the gas itself away from the electrodes, for this.would require not only molecular or atomic dissymmetry, but a fixed orientation of these particles. Hence we must regard rectification as due to conditions existing at the metal-gas surface and in the immediately adjacent metal and gas. The general form assumed by a single-phase alternating current wave when a conducting gas is included in the circuit is discussed later on in relation to the dissymmetry of the wave caused by partial rectification.

The greater part of the work here described was done with the current from a transformer of $100: 2,000$ volts and 600 watts capacity. Occasionally some of the work was repeated on smaller $100: 1,000$ volt transformers of 100 or 300 watts capacity. To control the current in the secondary a variable noninductive resistance of about 100 ohms was placed in the primary of the transformer. The current in 
the secondary varied from 10 to 40 milliamperes, according to the density of the conducting gas. Most of the work was done with ordinary Plücker tubes slightly altered according to the conditions desired. They were of the short, stout form now preferred and had either rod or disk aluminum electrodes. The tubes were connected with rubber pressure tubing to a Geryk pump and hydrogen generator by a threeway cock, so that they could be quickly exhausted and filled or washed

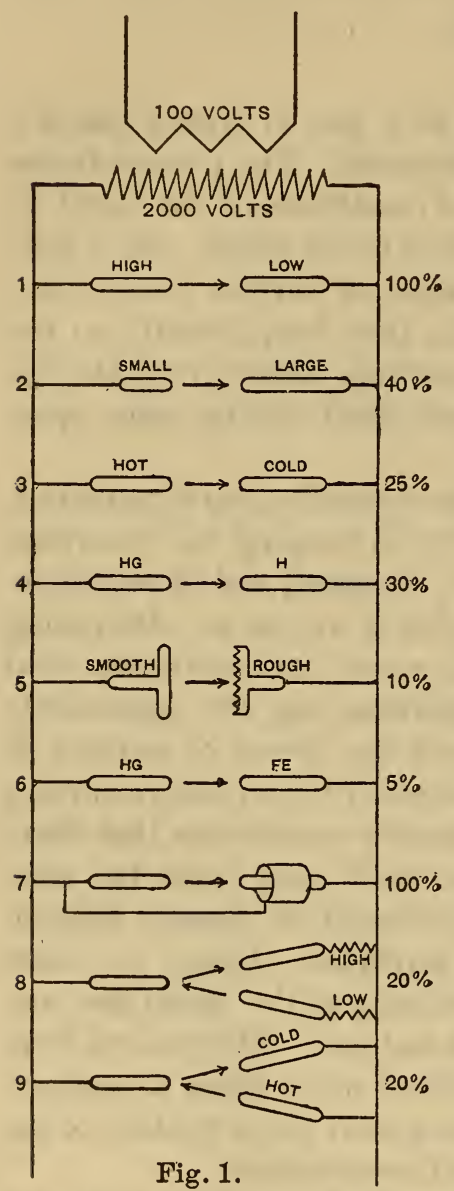
with hydrogen. A direct-current milli. ameter in series with the tube gave the amount of current rectified, while an alternating current volltmeter used in series as an ammeter, gave the total current. In this way rectification as small as half of one per cent could be easily measured and a tenth as much detected. It is difficult to prepare a tube that will not show as much as one per cent rectification. The effects here recorded are as large as 5 to 40 per cent and decidedly reversible.

The principal rectifying effects obtained are indicated diagrammatically in the accompanying figures. The number at the left corresponds with the number of the descriptive paragraph in the context. The percentages at the right indicate the maximum percentage of the current rectified, while the intermediate arrow indicates its direction. Except the first, the effects showed "but little variation in percentage of rectification with the density of the conducting gas, as the pressure on this was varied from 6 to $0.1 \mathrm{~mm}$.

1. The difference in pressure of the gas about the two electrodes was accomplished by constricting the tube to a fine capillary between the electrodes and rapidly exhausting. If the exhaustion is sufficiently rapid, complete rectification occurs, i. e., half of the current wave fails to pass at all. Judging by the width of the cathode dark space, the corresponding pressures in the two bulbs are about $4 \mathrm{~mm}$. and $0.3 \mathrm{~mm}$. Sudden admission of gas into an exhausted tube reverses the rectification. 
We can hardly attribute this effect to any differences within the electrodes nor to the ease of escape of electrons from metal to gas. But if we consider the current carried by the negative electrons alone, then the current will be able to start much more easily from the electrode surrounded by the more rarefied gas, for here the electrons will have a greater free path and will be more easily able to attain ionizing velocity. Hence the (negative) current will travel most easily from low to high pressure as observed. A cruder and less tenable explanation is possible if we consider the current carried by both positive and negative electrons. The strong current of gas through the capillary from high to low pressure would tend to sweep the larger positive electrons with it rather than the much smaller negative electrons. Hence the (positive) current would flow from high to low pressure as observed.

2. The relative size of the electrodes is an important factor in producing rectification. The excess of current flows from the small to the large electrode. When the large electrode was three times the area of the smaller, a rectification of 12 per cent was observed; when it was ten times the area, 35 per cent was observed. The tests were made with aluminum electrodes in Plücker tubes. When the pressure is so great and the current so small that the glow covers only a portion of the larger electrode the effect is null or reversed. After the current has been passing for some time the heating effect next described (3) may mask the effect of relative size. There appears to be little if any difference in size of the cathode dark space at the large and small electrodes.

The current density being less at the larger electrode, any competition between the electrons carrying the current will be less at this electrode. At the larger electrode negative electrons starting out would be less interfered with by the pressure of positive electrons. The effect can hardly be traced to the difference in capacity of the large and small electrodes, for connecting one electrode to a large capacity or even to earth produces but a slight rectifying effect.

3. A difference in temperature of the electrodes gives a partly rectified current from the hot to the cold electrode. When one bulb of a Plücker tube was heated to about $250^{\circ}$ and the other bulb kept cold by a wet cloth and protected by an asbestos screen, a rectification as large as 25 per cent was observed on starting the current. This decreased rapidly as the current heated the cold electrode. Using tubes with disk electrodes the cathode dark space was at least 50 per cent larger at the hot electrode than at the cold. At the same time the canal ray glow within the dark space was scarcely perceptible while at the cold electrode it nearly filled the cathode dark space.

18364-No. 1-05-7 
A continuous current heats the cathode much more energetically than the anode. Hence when an alternating current passes between a hot and a cold electrode we should expect the rectified portion to pass in such a direction as to heat the cold electrode more than the hot, and so produce equilibrium. This is what actually occurs. But the electron theory would lead us to expect a current from cold to hot. At the hot electrode negative electrons escape from the metal most easily. They have a longer free path to get started and produce ionization, and this movement is less obstructed by the presence of positive electrons. Hence we should expect a negative current from hot to cold, the reverse of that observed.

4. Gas about the electrode.-When one bulb of a Plücker tube is filled with mercury vapor and the other with air or hydrogen there is a decided rectifying effect from the mercury vapor toward the hydrogen. In spectroscopic work I have frequently observed complete rectification under similar conditions.

In some previous work on the spectra of mixed gases (this bulletin) it was shown that in a mixture, other things being equal, gases or vapors of great atomic weight appear to give always greater spectral energy than those of lower. If we take this to mean that they carry current more readily or are more readily ionized, we have a simple explanation for the above rectifying effect. The current is more easily started in the bulb containing heavier vapor so that half the alternating current wave is reduced in area according to the diagram discussed later.

5. The condition of the surface of the electrodes appears to determine a slight but decided rectifying effect. Aluminum disk electrodes were used, one polished and the other cut in ridges, like a file, with a sharp knife. An excess of current passed from the smooth to the rough electrode. Whether this effect was anything more than that due to a greater exposed surface could not be determined.

6. With one electrode mercury and the other iron of the same size, but a slight rectifying effect from mercury to iron was observed on starting the current. After the current had been running some time mercury vapor over the mercury electrode tended by (4) to increase the rectification, while the much hotter iron electrode by (3) tended to decrease it. It would appear that the action of the Cooper Hewitt rectifier on a three-phase current is largely due to the much greater surface exposed by the mercury electrode and the greater density of mercury vapor in its immediate neighborhood. If cathode drop of potential played an important rôle, we should have obtained greater rectification with the tube just described and a single-phase current. 
When aluminum was used against iron in atmospheres of air and hydrogen, a rectification of 20 per cent was again observed. When hydrogen was used, the rectification was just perceptibly less than in air.

7. Placing a metallic band about one electrode and connecting this band with the other electrode, a strong, often complete, rectification is produced, the current passing toward the electrode having an external band. It has frequently been noticed that placing the hand near a discharge tube steadies the discharge. An earthed metallic band about the tube near one electrode was much more effective in strengthening

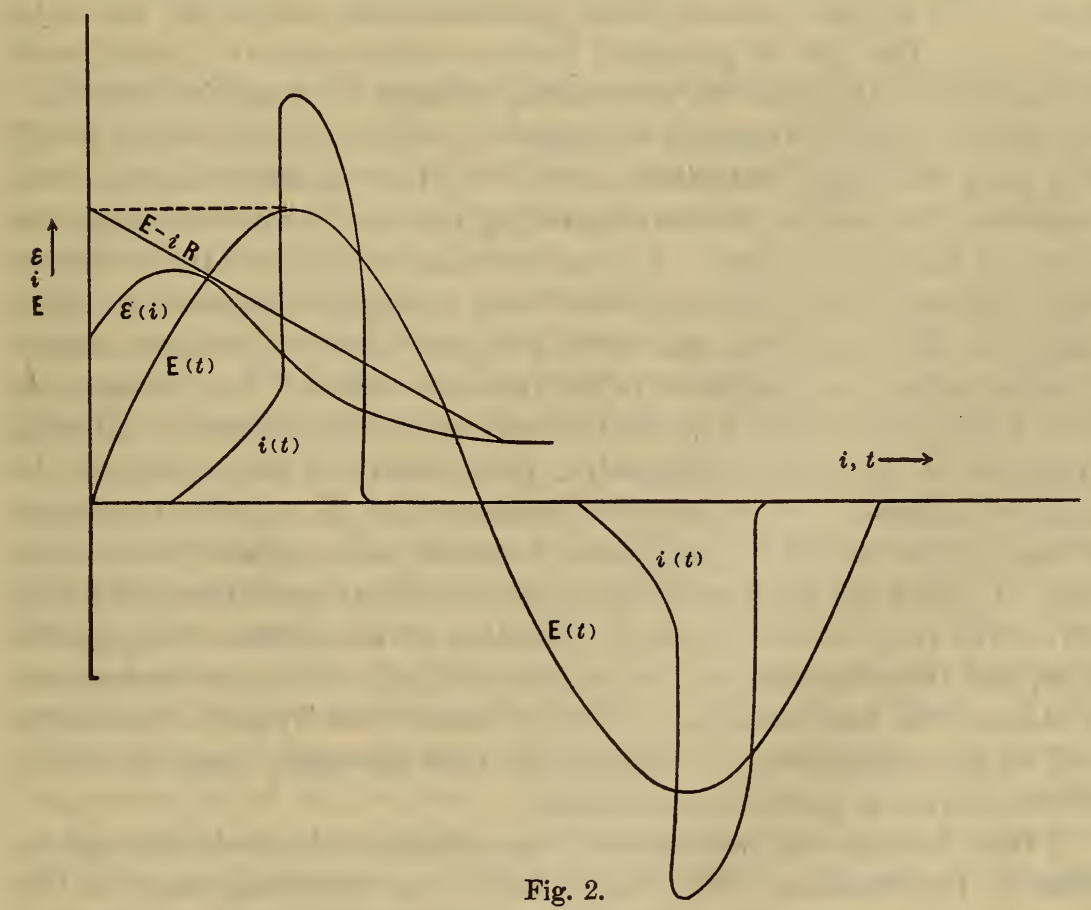

the discharge. Finally, this band was connected with the opposite electrode and complete rectification obtained. A similar device has been used to facilitate starting in mercury vapor lamps, a metallic covering being placed about the cathode and connected to the anode.

Throwing an intense beam of sunlight on the front face of one of the (disk) electrodes by means of a lens produced no perceptible rectification. The effect of exposing one electrode or the adjacent gas to the action of X rays was not tested, but from (4) and (6) we should expect the side exposed to become anode. 
Tests 8 and 9 were made with tubes provided with three mercury electrodes. One of these was connected to one 2,000-volt terminal, and the other two, through different resistances, to the other. Current was found to enter through the low resistance and leave by the high. Such an effect by (3) would be produced by the low-resistance electrode being more heated by the greater current. When connections were made with equal resistance and one electrode heated the current passed from hot to cold in accordance with (3) and (4).

We may get a better idea of what actually occurs in the process of partial rectification from the accompanying figure (fig. 2). Let the curve $\varepsilon(i)$ be the characteristic gas-conduction curve for the tube used-i. e., the fall of potential between electrodes as a function of the current. Impress the alternating voltage $\mathrm{E}(t)$ on the tube having such an external (ohmic) resistance in circuit that the line $\mathrm{E}-i \mathrm{R}$ will have the slope indicated, then $i(t)$, here plotted directly, will represent the form of the corresponding curve of current through the tube as a function of time. Anyone who has ever plotted these current curves knows how entirely their form changes with even a slight change in the form of the gas curve $\varepsilon(i)$, and these $\varepsilon(i)$ curves change in form greatly as conditions in the tube are varied. For instance, at very low pressures the $\varepsilon(i)$ curve is elevated and flattened. At high pressures it may rise continually, particularly if the electrodes be small or pointed. If the tube be illuminated by $\mathrm{X}$ rays the maximum of the $\varepsilon(i)$ curve will be sharpened, lowered, and displaced toward the left. If then the electrodes are under different conditions, the $\varepsilon(i)$ curve will vary in form with the direction of the current through the tube, and therefore the current curves $i(t)$ will no longer be symmetrical about the horizontal axis. The reversed (lower) part of the curve will be more flattened or rounded say than the upper, and the effect will be shown as partial rectification.

A tube having one internal and one external electrode showed no trace of rectification. This might have been expected, since in this case the $\varepsilon(i)$ curve rises steeply and continuously and the current curve $i(t)$ is a low, broad sine curve which would show little, if any, dissymmetry. 


